\title{
Study of Delayed Development of the Upper First Molars
}

\author{
By \\ Natsuki SANO ${ }^{1}$, Yuh HASEGAWA ${ }^{1}$, Shigeki IJJMA ${ }^{2}$ and Kazuto TERADA ${ }^{1}$ \\ ${ }^{1}$ Department of Orthodontics, The Nippon Dental University School of Life Dentistry at Niigata \\ ${ }^{2}$ Iijima Orthodontic Office in Mito city
}

- Received for Publication, February 18, 2010-

\begin{abstract}
Key Words: upper first molar, delayed development, logistic curve
Summary: The present study was undertaken to analyze the status of formation of the upper first molar in individuals suspected as having delayed eruption of the upper first molar.

The subjects of this study were 51 patients presenting to orthodontic specialists with delayed eruption of the upper first molars. The 95 teeth of these 51 patients were observed. The investigation of the tooth development status included evaluation of the tooth development stage on panoramic radiographs according to the method of Moorrees $\mathrm{et} \mathrm{al}$.

The upper first molars that showed delayed development were at the stage of initial cleft formation, reflecting a delay by 2.09 years in boys and 2.84 years in girls. In most cases, the delayed eruption was bilateral, and the development stage of the subject teeth did not differ between right and left sides.

The plot of the developmental stage of the subject teeth (Y-axis) against the calendar age of the subjects (X-axis) showing delayed upper first molar development was fitted to the logistic curve. The tooth development was at the stage of initial cleft formation was delayed 2.5 years or more, according to these curves.
\end{abstract}

\section{Introduction}

The first molar is considered as one of the key elements of occlusion and plays an important role as the center of occlusion. If an eruption of the first molars is disturbed for some reason, the compromised masticatory function and the absence of occlusion between the upper and lower first molars cause unstable occlusion, adversely affecting jaw development, etc.

Disturbed eruption of the permanent teeth has been reported to occur at an incidence of four percents ${ }^{1,2)}$. The disturbance often occurs on the upper and lower third molars, upper canines and lower second premolars, but rarely on the first molars ${ }^{2-4)}$. However, reports have also been published concerning disturbed eruption or congenital absence of the first molars ${ }^{5-15}$. Reported factors responsible for disturbed eruption of the first molars include systemic diseases, e.g., cleidocranial dysplasia, odontogenic tumor, odontoma, ectopic eruption and delayed tooth development ${ }^{9}$, 10). However, few reports have been published concerning the tooth development processes of the teeth showing delayed development.

The methods reported by Nolla ${ }^{16)}$ and Moorrees et $a l^{17)}$ were usually employed for evaluation of the status of tooth development. In these methods, the stages of crown and tooth root formation were scored on the basis of panoramic radiograph data. Demirjian et al. ${ }^{18,19)}$ reported a method for evaluating the systemic growth stage by applying the development stage scores of seven teeth with assessed according to the method of Moorrees et al. ${ }^{17)}$ to the logistic curve.

The logistic curve has the advantage that checking the fitness to this curve enables evaluation of the speed of development and the status of initiation of development. As the coefficient $\mathrm{A}$ in the logistic curve, defined as $\mathrm{Y}=$ $\mathrm{K} /\left(1+\mathrm{A} \cdot \mathrm{e}^{-\mathrm{B} \cdot \mathrm{X}}\right)$, becomes larger, the curve is shifted in the rightward direction, indicating delayed development. As coefficient B becomes larger, the sharper the initial segment of the curve becomes, reflecting more rapid development.

In view of the above-mentioned sporadic reports of delayed development of the first molars, it seems an important issue in the field of dentistry to investigate the delayed development of the first molars, taking into account the influence of disturbed eruption on occlusion.

The present study was undertaken to evaluate the status of formation of the upper first molar in individuals suspected as having delayed eruption of the upper first 
molar as compared with the standard timing of eruption in Japanese. In addition, this study was designed to evaluate the fitness of the data on the tooth development stage to a logistic curve.

\section{Subjects}

Of the patients visiting clinics specializing in orthodontics (Niigata City, Nagaoka City and Mito City), boys and girls aged over 6 years were enrolled in this study, excluding the following cases: patients rated on X-ray as having abnormal eruption of the upper first molars for reasons such as ectopic eruption or odontoma and patients having systemic diseases including cleft of the lip, jaw and palate. The standard timings of the eruption of the upper first molar in Japanese were reported to be 6 years 8 months (and 8 months as SD.) in boys, 6 years 7 months (and 8 months as SD.) in girls ${ }^{20,21}$. The subjects of this study were 51 patients presenting with delayed eruption of the upper first molars, i.e., those in whom it took two-fold or more time for eruption of the upper first molars as compared to the standard timing of eruption in Japanese and in whom the germ or the like for the upper third molars remained absent during the subsequent follow-up period. The 95 teeth of these 51 patients were observed in this study. Seven patients were appeared unilateral, and 44 patients were bilateral (Table 1, Fig. 1). There were three patients who had upper third molars despite the delayed eruption of the upper first molars; however, to make the conditions uniform for all subjects, the three patients having upper third molars were excluded from the study.

In this study, the data were obtained from panoramic radiographs taken for orthodontic treatment. The age and gender of each subject were recorded. The data were converted into non-linkable anonymous information prior to the start of the analysis.

\section{Methods}

In view of the finding reported by Mitomi et $a .^{21)}$, who investigated delay in eruption of the first molars in the Japanese population, we investigated by gender the location and development status of the upper first molars showing delayed eruption, i.e., taking two-fold or more time for eruption as compared with the standard timing for Japanese $\mathrm{e}^{20)}$. Evaluations were performed twice by the same examiner at an interval of 1 month or more.

Table 1. Classification of subjects

\begin{tabular}{|c|c|c|c|c|c|c|c|c|c|c|c|c|}
\hline & \multicolumn{4}{|c|}{ Male } & \multicolumn{4}{|c|}{ Female } & \multicolumn{4}{|c|}{ Total } \\
\hline & unilateral & bilateral & $\begin{array}{c}\text { total } \\
\text { (persons) }\end{array}$ & $\begin{array}{c}\text { total } \\
\text { (teeth) }\end{array}$ & unilateral & bilateral & $\begin{array}{c}\text { total } \\
\text { (persons) }\end{array}$ & $\begin{array}{c}\text { total } \\
\text { (teeth) }\end{array}$ & unilateral & bilateral & $\begin{array}{c}\text { total } \\
\text { (persons) }\end{array}$ & $\begin{array}{c}\text { total } \\
\text { (teeth) }\end{array}$ \\
\hline A orthodontic office & 0 & 3 & 3 & 6 & 0 & 10 & 10 & 20 & 0 & 13 & 13 & 26 \\
\hline B orthodontic office & 0 & 5 & 5 & 10 & 1 & 12 & 13 & 25 & 1 & 17 & 18 & 35 \\
\hline C orthodontic office & 3 & 7 & 10 & 17 & 3 & 7 & 10 & 17 & 6 & 14 & 20 & 34 \\
\hline
\end{tabular}

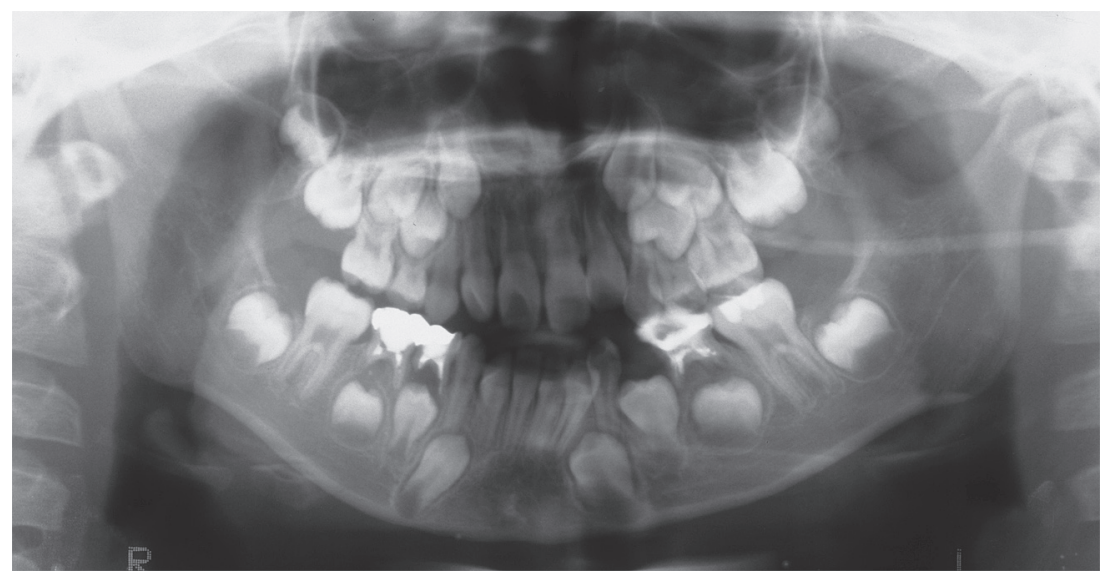

Fig. 1. Delayed development of upper first molars on panoramic radiograph (9y1m, female). 


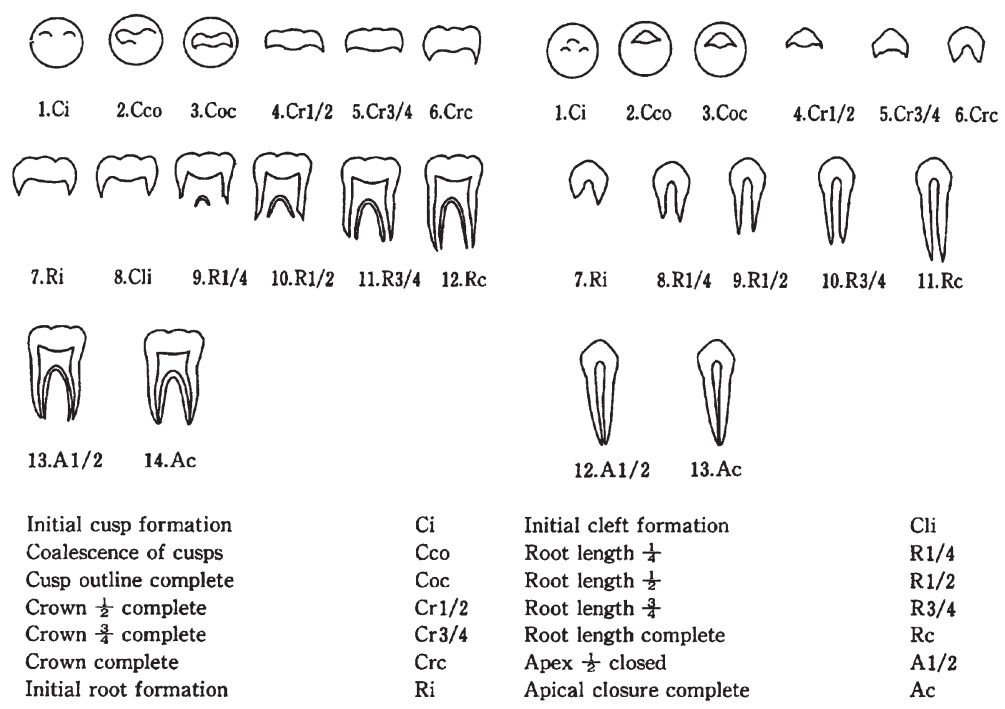

Fig. 2. Tooth formation stages according to Moorrees et al.

The development status of the upper first molars was evaluated on a 14-stage scale in accordance with the criteria for assessment of the development stages proposed by Moorrees et al. ${ }^{17)}$ (Fig. 2). The ipsilateral lower canines were rated on a 13-stage scale as the control group. The lower canines were selected as the control group, because they are relatively stable as compared to the other teeth and the impaction is relatively more likely to occur with the upper canines.

On the basis of the data obtained, we checked the difference between right and left sides and gender-associated differences in the development stage of the teeth, and any differences observed were tested statistically by the Mann-Whitney test.

Then, the plot of the tooth development stage (Y-axis) against the calendar age of the subjects (X-axis) was fitted to a logistic curve, and the degree of fitness was rated at a significance level of $5 \%$. The coefficients A and $\mathrm{B}$ for the significantly fitting logistic curve were analyzed.

\section{Results}

\section{Location and development stage}

Among the 51 patients enrolled in this study (18 males and 33 females), the delayed eruption was unilateral in 7 patients (14\%) and bilateral in 44 patients $(86 \%)$. The 95 upper first molars and the lower canines as the control teeth of the 51 patients were assessed to determine the development status using panoramic radiographs taken over years, in accordance with the criteria for assessment of the development stage proposed by Moorrees et al. ${ }^{17}$

There was no difference of the development statues between right and left sides in the lower canine of subject persons as the control teeth. Therefore, the data of the left lower canines were included for the analysis. Tooth formation stages on the lower canines were beyond "crown $3 / 4$ complete" in all of the boys and beyond "crown complete" in all of the girls. There was one boy aged 7.67 years old in whom the stage was "crown complete." The stage "initial root formation" was observed in three boys (average age $=6.50$ years, $\mathrm{SD} .=0.43$ years) and five girls (average age $=6.74$ years, SD. $=0.65$ years). The stage "root length 3/4" was observed in 12 boys (average age $=10.49$ years, $\mathrm{SD} .=0.75$ years) and 20 girls (average age $=9.51$ years, $\mathrm{SD} .=0.76$ years) $($ Table 2$)$.

For the subject teeth, data before the stage of "crown $3 / 4$ complete" were unavailable. The stage "crown complete" was observed for one tooth of one boy (age, 7.67 years) and two teeth of two girls (average age $=6.83$ years). The stage "initial cleft formation" was observed for 4 teeth among the boys (average age $=6.67$ years, $\mathrm{SD} .=0.83$ years) and 24 teeth among the girls (average age $=7.46$ years, $\mathrm{SD} .=0.87$ years). The stage "root length complete" was observed for 15 teeth among the boys (average age $=10.60$ years, $\mathrm{SD} .=1.26$ years) and 21 teeth among the girls (average age $=10.32$ years, SD. $=1.37$ years) $($ Table 3$)$.

\section{Laterality and gender-associated differences in the development stage}

No laterality in tooth development was noted in the patients showing bilateral delay in tooth eruption; there were no gender-associated differences in terms of the development stage of any of the teeth evaluated. 
Table 2. Tooth development; this study and Japanese average values on lower canines

( yrs)

\begin{tabular}{|c|c|c|c|c|c|c|c|c|c|c|c|c|c|c|c|c|}
\hline & & & & & & Mal & & & & & & & Femal & & & \\
\hline & & & & & & & & Japanese & values & & & & & & Japanese & values \\
\hline & 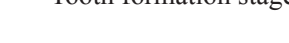 & & & & His siu & & & Niimura & 22) & & & & & & Niimur & 22) \\
\hline & & & $\mathrm{N}$ & Average & SD & Min & Max & Average & SD & $\mathrm{N}$ & Average & SD & Min & Max & average & $\mathrm{SD}$ \\
\hline 1 & Initial cusp formation & (Ci) & & & & & & & & & & & & & & \\
\hline 2 & Coalescence of cusps & $(\mathrm{Cco})$ & & & & & & & & & & & & & & \\
\hline 3 & Cusp outline complete & (Coc) & & & & & & & & & & & & & & \\
\hline 4 & Crown $1 / 2$ complete & $(\mathrm{Cr} 1 / 2)$ & & & & & & 3.69 & 0.63 & & & & & & 3.38 & 0.38 \\
\hline 5 & Crown $3 / 4$ complete & $(\mathrm{Cr} 3 / 4)$ & & & & & & 4.57 & 1.01 & & & & & & 4.24 & 0.93 \\
\hline 6 & Crown complete & $(\mathrm{Crc})$ & 1 & 7.67 & & 7.67 & 7.67 & 5.57 & 0.98 & & & & & & 5.00 & 1.01 \\
\hline 7 & Initial root formation & (Ri) & 3 & 6.50 & 0.43 & 6.25 & 7.00 & 6.40 & 1.15 & 5 & 6.74 & 0.65 & 6.00 & 7.92 & 6.36 & 1.03 \\
\hline & Root length $1 / 4$ & (R1/4) & 13 & 8.22 & 0.70 & 6.33 & 9.00 & 7.73 & 1.26 & 22 & 7.36 & 0.73 & 6.00 & 9.08 & 7.37 & 1.18 \\
\hline & Root length $1 / 2$ & (R1/2) & 17 & 9.03 & 0.73 & 7.58 & 10.00 & 8.80 & 1.31 & 26 & 8.76 & 1.61 & 7.00 & 16.00 & 8.13 & 1.04 \\
\hline 10 & Root length $3 / 4$ & (R3/4) & 12 & 10.49 & 0.75 & 9.17 & 12.00 & 10.12 & 1.13 & 20 & 9.51 & 0.76 & 7.67 & 10.75 & 9.23 & 1.25 \\
\hline 11 & Root length complete & $(\mathrm{Rc})$ & 10 & 11.26 & 1.15 & 9.00 & 13.00 & 11.28 & 1.26 & 19 & 10.40 & 0.91 & 8.00 & 11.92 & 10.26 & 1.08 \\
\hline 12 & Apex $1 / 2$ closed & $(\mathrm{A} 1 / 2)$ & 10 & 12.42 & 1.08 & 11.00 & 14.00 & - & - & 14 & 11.60 & 0.66 & 10.00 & 12.67 & - & - \\
\hline 13 & Apical closure complete & $(\mathrm{Ac})$ & 8 & 13.70 & 0.95 & 12.42 & 15.00 & - & - & 15 & 13.21 & 1.20 & 11.92 & 16.00 & - & - \\
\hline
\end{tabular}

Table 3. Tooth development of the delayed upper first molars; this study and Japanese average values

( yrs)

\begin{tabular}{|c|c|c|c|c|c|c|c|c|c|c|c|c|c|c|c|c|}
\hline & & & & & & Mal & & & & & & & Fema & & & \\
\hline & Touth formation stas & & & & his stu & & & Japanese & values & & & his stu & & & Japanese & values \\
\hline & & & & & & & & Niimura & 22) & & & & & & Niimur & 22) \\
\hline & & & $\mathrm{N}$ & Average & SD & Min & Max & Average & SD & $\mathrm{N}$ & Average & SD & Min & Max & Average & SD \\
\hline 1 & Initial cusp formation & (Ci) & & & & & & & & & & & & & & \\
\hline & Coalescence of cusps & $(\mathrm{Cco})$ & & & & & & & & & & & & & & \\
\hline & Cusp outline complete & $(\mathrm{Coc})$ & & & & & & & & & & & & & & \\
\hline 4 & Crown $1 / 2$ complete & $(\mathrm{Cr} 1 / 2)$ & & & & & & & & & & & & & & \\
\hline 5 & Crown $3 / 4$ complete & $(\mathrm{Cr} 3 / 4)$ & & & & & & 3.42 & 0.48 & & & & & & 3.36 & 0.38 \\
\hline 6 & Crown complete & $(\mathrm{Crc})$ & 1 & 7.67 & & 7.67 & 7.67 & 3.83 & 1.18 & 2 & 6.83 & 0.00 & 6.83 & 6.83 & 3.78 & 0.56 \\
\hline 7 & Initial root formation & (Ri) & 2 & 7.00 & 0.00 & 7.00 & 7.00 & 4.19 & 0.91 & 2 & 7.50 & 0.00 & 7.50 & 7.50 & 3.89 & 0.73 \\
\hline & Initial cleft formation & (Cli) & 4 & 6.67 & 0.83 & 6.25 & 7.92 & 4.58 & 0.79 & 24 & 7.45 & 0.87 & 6.33 & 8.83 & 4.61 & 1.20 \\
\hline 9 & Root length $1 / 4$ & (R1/4) & 19 & 8.33 & 0.93 & 6.33 & 9.92 & 5.66 & 1.31 & 38 & 8.11 & 0.96 & 6.33 & 10.00 & 5.40 & 1.18 \\
\hline 10 & Root length $1 / 2$ & $(\mathrm{R} 1 / 2)$ & 19 & 9.15 & 0.78 & 7.92 & 10.50 & 6.13 & 0.87 & 39 & 8.74 & 1.13 & 6.00 & 11.00 & 6.37 & 1.12 \\
\hline 11 & Root length $3 / 4$ & (R3/4) & 18 & 9.71 & 1.29 & 7.83 & 11.75 & 7.53 & 1.08 & 43 & 9.85 & 1.40 & 6.92 & 11.92 & 7.39 & 0.93 \\
\hline 12 & Root length complete & $(\mathrm{Rc})$ & 15 & 10.60 & 1.26 & 8.92 & 12.42 & 8.97 & 1.15 & 21 & 10.32 & 1.37 & 8.00 & 13.00 & 8.74 & 1.19 \\
\hline 13 & Apex $1 / 2$ closed & $(\mathrm{A} 1 / 2)$ & 11 & 10.91 & 1.30 & 9.00 & 12.08 & 9.88 & 1.07 & 15 & 11.40 & 1.68 & 8.00 & 13.00 & 9.73 & 0.91 \\
\hline 14 & Apical closure complete & $(\mathrm{Ac})$ & 13 & 12.29 & 1.30 & 10.00 & 14.00 & 10.86 & 0.71 & 35 & 12.91 & 1.93 & 10.00 & 16.00 & 10.14 & 1.14 \\
\hline
\end{tabular}

\section{Fitness to logistic curve}

The plot of the development stage according to Moorrees' criteria $^{17)}$ of the subject teeth (Y-axis) against the calendar age of the subjects (X-axis) showing was fitted to a logistic curve defined by $Y=K /\left(1+A \cdot e^{-B \cdot X}\right)$. In this analysis, the values of coefficient $\mathrm{A}$ and $\mathrm{B}$ were 54.845 and 0.541 , respectively, in boys and 51.131 and 0.529 , respectively, in girls. Thus, fitness to the logistic curve was shown ( $<0.05$ for both boys and girls) (Figs. 3 and 4).

\section{Discussion}

Location of delayed eruption and development status of teeth

Investigation of 51 patients showing delayed eruption of the upper first molars revealed that the delayed eruption was unilateral in seven cases (14\%) and bilateral in 44 cases $(86 \%)$. Thus, bilateral delay of eruption was clearly more frequent than unilateral one. There were no gender-associated differences, endorsing the findings of Rassmussen ${ }^{9}$.

In regard to the nature of delayed eruption of the upper first molars, Rasmussen ${ }^{9)}$ reported that the delayed 


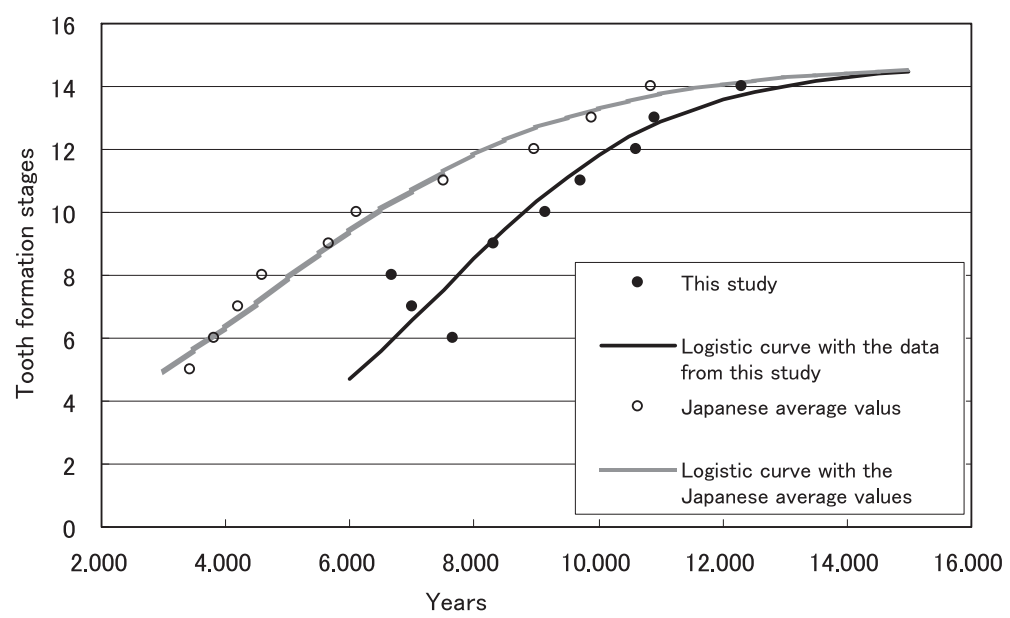

Fig. 3. Logistic curves of delayed development of the upper first molars (male).

Female

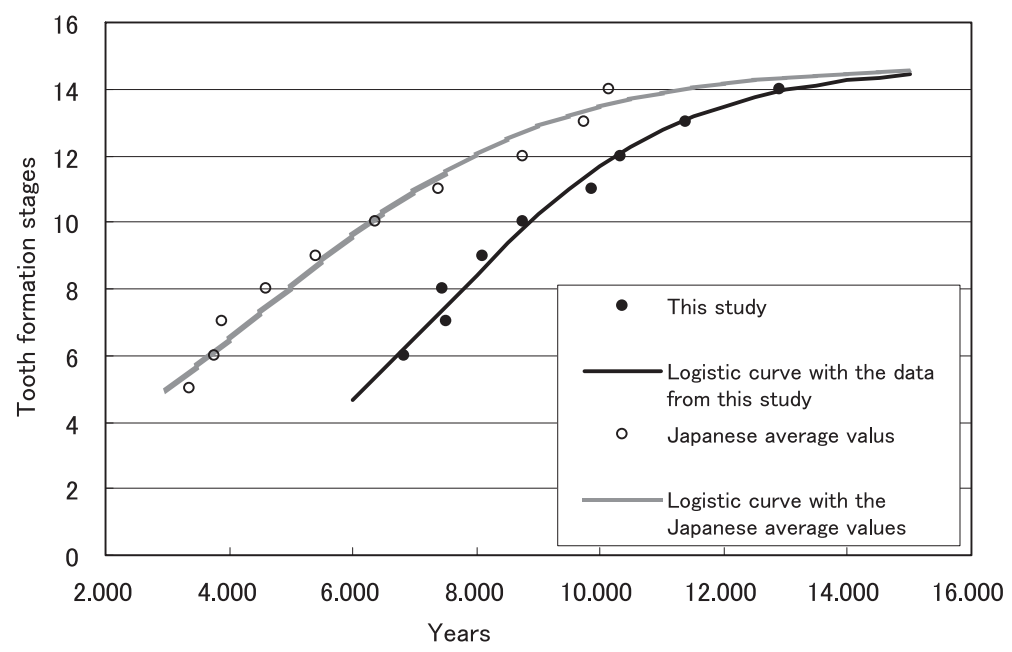

Fig. 4. Logistic curves of delayed development of the upper first molars (female).

eruption was unilateral in only 1 of the 10 cases. In the present study also, the number of cases showing unilateral delay was relatively very small. According to Rasmussen ${ }^{9}$, four cases had at least one brother or sister showed delay in eruption of the upper first molars. In our study, none of the patients had any brothers or sisters showing delayed tooth eruption.

Analysis of the lower canine as the control teeth in our study revealed that the development of the root was slower as compared to the mean timing of root development reported by Niimura ${ }^{22)}$. However, the difference was less than one standard deviation, indicating that the development of the lower canine roots occurred at ap- proximately the average age reported for Japanese. In the analysis of the upper first molars, the recognition of the "initial cleft formation" stage was delayed 2.09 years in boys and 2.84 years in girls, and that of the "root length complete" stage was delayed 1.03 years in boys and 1.58 years in girls (Table 3). Thus, delayed development of the upper first molars began to be observed even from the early stages of development, the delay becoming less obvious at the "root length complete" stage, suggesting that the root development of these teeth was relatively rapid.

When the results for the average development stages for permanent teeth in Japanese reported by Niimura ${ }^{22}$ 
were compared with the data on standard deviation obtained in our study, the standard deviation for the upper first molars was smaller for the stages of "root length $1 / 4$ " and "root length $1 / 2$ " in the boys and for the stages of "initial cleft formation" and "root length $1 / 4$ " in the girls. For the subsequent development stages, the standard deviation in our study was greater for both males and females. These results suggested that the timing of formation of these teeth is also uniform, as seen for ordinary teeth.

Gender-associated differences in the mean tooth development stages have been reported in Japanese $\mathrm{e}^{22)}$. In our study, however, no significant gender-associated differences were observed, although the development was slightly delayed in boys.

\section{Fitting to the logistic curve and comparison with mean} growth for Japanese

Analysis of changes in the growth of organisms by determining their fitting to logistic curves has been reported to be a valid approach. Terada et al. ${ }^{23)}$ reported good fitting to the logistic curves in relation to the length of the finger bones and the mandible. Ever since Demirjian et al. ${ }^{18,{ }^{19)}}$ reported a method for evaluation of the systemic growth stage on the basis of the fitting of the tooth development state to the logistic curve, numerous reports on the clinical application of this method have been published ${ }^{24,25}$. According to the reports on tooth development, including the reports by Nolla ${ }^{16)}$ and Moorrees et $a{ }^{177}{ }^{17}$, inter-individual variations in tooth development are small, and tooth development shows close correlation with the calendar age in a stable manner.

We fitted the Japanese data using the criteria of Moorrees reported by Niimura ${ }^{22)}$ to the logistic curve. In this analysis, fitness to the logistic curve was noted at a significance level of $5 \%$ for both males and females (Figs. 3 and 4). We then compared the "initial cleft formation" stage with the "root length complete" stage on the basis of the data obtained from the present study and the logistic curve yielded from the average data for Japanese. The "initial cleft formation" stage fitting the logistic curve was at the age of 7 years 9 months for both boys and girls in the present study, while it was 5 years 1 month for boys and 4 years 11 months for girls according to the data reported by Niimura ${ }^{22)}$. The "root length complete" stage was at age 10 years 2 months for boys and 10 years 3 months for girls in our study, whereas it was 8 years 2 months for boys and 7 years 11 months for girls according to the data reported by Niimura ${ }^{22)}$. Thus, the development stage in the children studied by us was delayed approximately 2.5 years or more. Rasmussen used the expression "9-year-molars" to indicate the molars whose development was delayed as compared to the average. The delay observed in our study was similar to that reported by Rasmussen ${ }^{9}$.

\section{Perspectives for the future}

The present study was designed to investigate delay in tooth development. In studies aimed at investigating rare abnormalities like delayed tooth development, collection of data is important. Data from only one facility will not suffice, and participation of multiple facilities would be desirable. Conducting this kind of the studies of more extensive data may be expected to make highly precise evaluation of the tooth development status possible. Therefore, we propose the need for collecting further data.

The present study observed the molars located posterior to the upper deciduous second molars in dentitions, without the third molar. This study did not incorporate any distinction between first and second molars. According to the results of this study, the teeth investigated were at an intermediate stage between the development stage of the first and second molars. In this connection, Bolk ${ }^{26}$ reported that congenital defects takes place at a point distal to this type of tooth. There is no consensus among investigators over congenital defects or delayed eruption of the first molars. It would be desirable to adopt some additional methods for morphological judgment of teeth. This is another open issue that needs to be resolved for clarification of delayed eruption.

\section{Conclusion}

We evaluated the development status of the teeth in patients suspected to have delayed eruption of the upper first molars, using the criteria for assessment of the development stage proposed by Moorrees et al. The subjects of this study were 51 patients who presented with delayed eruption of the upper first molars, i.e., those in whom it took two-fold or more time for eruption of the upper first molars as compared with the standard timing of eruption in Japanese, and in whom the germ or the like for the upper third molars remained absent during the subsequent follow-up period. The 95 teeth of these 51 patients were studied. And seven cases were delayed unilateral, 44 cases bilateral.

Delayed eruption was rarely unilateral and in most cases bilateral, and there was no difference between right and left sides in the development state in the bilateral cases.

Among the upper first molars showing delayed eruption, the average age of recognition of the "initial cleft formation" stage was 6.67 years for boys and 7.46 years for girls, and that of the "root length complete" stage was 10.60 years for boys and 10.32 years for girls. There was no laterality in the development stage of the upper first molars showing delayed eruption.

The plot of the rating by the Moorrees' development stage assessment criteria against the calendar age of the subjects was found to fit the logistic curve well, indicat- 
ing that the "initial cleft formation" stage of the patients examined was delayed 2.5 years or more as compared to the average for Japanese.

\section{Acknowledgments}

We are indebted to Dr. Masato Ohtake (Orthodontist in Nagaoka city, Niigata prefecture) and Dr. Michio Sugiyama (Orthodontist in Niigata city) for their data and their advice on this study.

\section{References}

1) Johnsen DC. Prevalence of delayed emergence of permanent teeth as a result of local factors. JADA 1977; 94:100-106.

2) Noda T, Takagi M, Hayashi-Sakai S and Taguchi Y. Eruption disturbances in Japanese children and adolescents. Pediatric Dental J 2006; 16:50-56.

3) Kramer RM and Williams AC. The incidence of impacted teeth. Oral Surg 1970; 29:237-241.

4) Grover PS and Lorton L. The incidence of unerupted permanent teeth and related clinical cases. Oral Surg 1985; 59:420-425.

5) Philipsen HP, Thosaporn W, Reichart PA and Grundt G. Odontogenic lesions in opercula of permanent molars delayed in eruption. J Oral Pathol Med 1982; 21:38-41.

6) Bjerklin K, Gleerup A and Kurol J. Long-term treatment effects in children with ectopic eruption of the maxillary first permanent molars. Eurp J Orthod 1995; 17:293-304.

7) Chintakanon K. Ectopic eruption of the first permanent molars: Prevalence and etiologic factors. Angle Orthod 1998; 68:153160.

8) Tomizawa M, Yonemochi H, Kohno $\mathrm{M}$ and Noda T. Unilateral delayed eruption of maxillary permanent first molars: four case reports. Pediatr Dent 1998; 20:53-56.

9) Rasmussen P. 9-year-molars aberrantly developing and erupting: report of cases. J Clin Pediatr Dent 1998; 22:151-153.

10) Nakao K, Matsuoka T, Takahashi A, Matsumura M, Sobue $S$ and Ooshima T. Delayed development or congenital absence of a single first permanent molar in Japanese child patients. Int $\mathrm{J}$ Paediatric Dent 1999; 9:271-276.

11) Valmaseda-Castellón E, De-la-Rosa-Gay $\mathrm{C}$ and Gay-Escoda C.
Eruption disturbances of the first and second permanent molars: Results of treatment in 43 cases. Am J Orthod Dentofacial Orthop 1999; 116:651-658.

12) Baccetti $T$. Tooth anomalies associated with failure of eruption of first and second permanent molars. Am J Orthod Dentofacial Orthop 2000; 118:608-610.

13) Barberia-Leache E, Suarez-Clúa $C$ and Saavedra-Ontiveros D: Ectopic eruption of the maxillary first permanent molar: Characteristics and occurrence in growing children. Angle Orthod 2005; 75:610-615.

14) Becktor KB, Steiniche K and Kjær I. Association between ectopic eruption of maxillary canines and first molars. Eurp J Orthod 2005; 27:186-189.

15) Proff $P$, Bayerlein T, Fanghänel J, Allegrini S Jr and Gedrange T. Morphological and clinical considerations of first and second permanent molar eruption disorders. Ann Anat 2006; 188:353361.

16) Nolla CM. The development of the permanent teeth. J Dent Child 1960; 27:254-266.

17) Moorrees CFA, Fanning EA and Hunt EE. Age variation of formation stages for ten permanent teeth. J Dent Res 1963; 42:14901502.

18) Demirjian A, Goldstein $H$ and Tanner JM. A new system of dental age assessment. Hum Biol 1973; 45:211-227.

19) Demirjian A and Goldstein H. New system for dental maturity based on seven and four teeth. Ann Hum Biol 1976; 3:411-421.

20) The Japanese Society of Pedodontics. The chronology of deciduous and permanent dentition in Japanese children. Jpn J Ped Dent 1988; 26:1-18.

21) Mitomi T, Tomizawa $M$ and Noda T. A study of delayed eruption of the permanent first molar and its management for eruption and alignment, Jpn J Ped Dent 2000; 38:1080-1090.

22) Niimura K. Development of permanent tooth formation in children (in Jpn). J Fukuoka Dent Coll 1995; 22:485-509.

23) Terada K, Saito I, Liu H and Hanada K. Longitudinal study on the length change of digital bones and mandible with a logistic curve. J Jpn Orthod Soc 1995; 54:413-424.

24) Goya HA, Satake T and Maeda T. Dental age in Japanese children using a modified Demirjian method. Pediatric Dental J 2009; 19: 82-88.

25) Campbell R, Weinshel R, Backeljauw P, Wilson S, Bean J and Shao M. Dental development in children with growth hormone insensitivity syndrome: Demirjian analysis of serial panoramic radiographs. Cleft Palate-Craniofacial J 2009; 46: 409-413.

26) Bolk L. Odontological essays. J Anat. 1922; 56:107-136. 